\title{
End-to-End Multimodal Emotion Recognition using Deep Neural Networks
}

\author{
Panagiotis Tzirakis, George Trigeorgis, Mihalis A. Nicolaou, Björn W. Schuller, and Stefanos Zafeiriou
}

\begin{abstract}
Automatic affect recognition is a challenging task due to the various modalities emotions can be expressed with. Applications can be found in many domains including multimedia retrieval and human computer interaction. In recent years, deep neural networks have been used with great success in determining emotional states. Inspired by this success, we propose an emotion recognition system using auditory and visual modalities. To capture the emotional content for various styles of speaking, robust features need to be extracted. To this purpose, we utilize a Convolutional Neural Network (CNN) to extract features from the speech, while for the visual modality a deep residual network (ResNet) of 50 layers is used. In addition to the importance of feature extraction, a machine learning algorithm needs also to be insensitive to outliers while being able to model the context. To tackle this problem, Long Short-Term Memory (LSTM) networks are utilized. The system is then trained in an end-to-end fashion where - by also taking advantage of the correlations of each of the streams - we manage to significantly outperform, in terms of concordance correlation coefficient, traditional approaches based on auditory and visual handcrafted features for the prediction of spontaneous and natural emotions on the RECOLA database of the AVEC 2016 research challenge on emotion recognition.
\end{abstract}

Index Terms - end-to-end learning, emotion recognition, deep learning, affective computing

\section{INTRODUCTION}

$\mathbf{E}$ MOTION recognition is an essential component towards complete interaction between human and machine, as affective information is fundamental to human communication. Applications of emotion recognition can be found in different domains. For instance, emotion states can be used to monitor and predict fatigue state [1]. In speech recognition, emotion recognition can be used in call centers, where the goal is to detect the emotional state of the caller and provide feedback for the quality of the service [2].

The task of recognizing emotions is challenging because human emotions lack of temporal boundaries and different individuals express and perceive emotions in different ways [3]. Although current work around emotion recognition was mostly concentrated around inferring the emotion of a subject out of its speech, other modalities such as visual information (facial gestures) have also been used.

P. Tzirakis and G. Trigeorgis are with the Department of Computing, Imperial College London, UK.

E-mail: panagiotis.tzirakis12@imperial.ac.uk

M. A. Nicolaou is with the Department of Computing, Goldsmiths, University of London, UK.

B. W. Schuller is with GLAM - Group on Language, Audio \& Music, Imperial College London, UK and Chair of Embedded Intelligence for Health Care and Wellbeing, University of Augsburg, Germany.

S. Zafeiriou is with Department of Computing, Imperial College London, UK and Center for Machine Vision and Signal Analysis, University of Oulu, Finland.
With the advent of deep neural networks in the last decade, a number of groundbreaking improvements have been observed in several established pattern recognition areas such as object, speech and speaker recognition, as well as in combined problem solving approaches, e.g., in audio-visual recognition, and in the rather recent field of paralinguistics.

Numerous studies have shown the favorable property of these network variants to model inherent structure contained in the speech signal [4], with more recent research attempting end-to-end optimization utilizing as little human a-priori knowledge as possible [5]. Nevertheless, the majority of these works make use of commonly hand-engineered features such as Mel-Frequency Cepstral Coefficients (MFCC), Perceptual Linear Prediction (PLP) coefficients, and supra-segmental features such as those used in the series of the ComParE [6] and the AVEC challenges [7], which build upon knowledge gained in decades of auditory research and have shown to be robust for many speech domains.

Recently, however, a trend in the machine learning community has emerged towards deriving a representation of the input signal directly from raw, unprocessed data. The motivation behind this idea is that, ultimately, the network learns an intermediate representation of the raw input signal automatically that better suits the task at hand and hence leads to improved performance.

In this paper, we study automatic affect sensing using both speech and visual information in an end-to-end manner. Features are extracted from the speech signal using a CNN architecture designed for the audio channel and from the visual information using a ResNet-50 network architecture [8]. The output of these networks are fused together and fed to an LSTM to find the affective state of individuals. Contrary to the current practices, where each network is trained individually and the results are simply fed to a subsequent classifier, our system is trained in an end-to-end manner. To our knowledge this is the first work in literature that applies such an endto-end model for an audio-visual emotion recognition task. Furthermore, we suggest using explicit maximization of the concordance correlation coefficient $\left(\rho_{c}\right)$ [9] in our model and show that this improves performance in terms of emotion prediction compared to optimizing the mean square error objective, which is traditionally used. Finally, by further studying the activations of different cells in the recurrent layers, we find the existence of interpretable cells, which are highly correlated with several prosodic and acoustic features that were always assumed to convey affective information in speech, such as the loudness and the fundamental frequency. A preliminary version of this work was presented in [10], where only the 
raw speech waveform was used. We extend this work by considering also the visual modality in an end-to-end manner.

To show the benefit of our proposed multimodal model, we evaluated it in the REmote COLlaborative and Affective (RECOLA) database. A part of this database was used for the Audio/Visual Emotion Challenge and Workshop (AVEC) 2016. Our model is trained and tested using the whole database. Results show that the multimodal model benefits from the two modalities by producing equal results for arousal and valence as the speech and visual networks, respectively. We compare the unimodal and the multimodal models using results obtained in the AVEC 2016 challenge. Only the papers that used the audio, visual or audiovisual modalities are considered. In order to perform a fair comparison we apply the proposed method on the test set of the AVEC challenge. As shown by our experiments, our unimodal models produce the best results for both the speech and visual modalities.

The remainder of the paper is organized as follows. Section II reports related studies on emotion recognition using multiple modalities with DNNs. Section III introduces the multimodal model architecture. Section IV describes the dataset used for the experiments. Section V presents the experiments performed and reports the results. Finally, section VI concludes this paper.

\section{RELATED WORK}

The performance of pattern recognition models has been greatly improved with DNNs. Recently, a series of new neural network architectures have been revitalized, such as autoencoder networks [11], Convolutional Neural Networks (CNNs) [12], Deep Belief Networks (DBNs) [13] or memory enhanced neural network models such as Long Short-Term Memory (LSTM) [14] models.

These models have been used in various ways for multimodal recognition tasks such as in speech recognition. For instance, Ngiam et al. [15] proposed a Multimodal Deep Autoencoder (MDAE) network to extract features from audio and video modalities. First, a bimodal DBN was trained to initialize the deep autoencoder and then the MDAE was finetuned to minimize the reconstruction error of both modalities. In another study, $\mathrm{Hu}$ et al. [16] proposed a temporal multimodal network named Recurrent Temporal Multimodal Restricted Boltzmann Machine (RTMRBM) to model audiovisual sequences of data. Another task that DNNs have also been used for is gesture recognition. In [17], the authors use skeletal information and RGB-D images to recognize gestures. More particularly, they use DBNs to process skeleton features and a 3D CNN for the RGB-D data. Temporal information is considered by stacking a Hidden Markov Model (HMM) on top.

The emotion recognition domain has highly benefited with the advent of DNNs. Some works explore deep learning approaches for speech emotion recognition. For instance, Han et al. [18] uses hand-crafted features to feed a DNN that produces a probability distribution over categorical emotion states. From these probabilities they compute statistics from the whole utterance and finally, they perform classification by training an extreme learning machine. Lim et al. [19] after transforming the data using short time Fourier transform, they used CNNs to extract high-level features. In order to capture the temporal structure LSTMs were used. In a similar work, Trigeorgis et al. [10] proposed an end-to-end model that uses a CNN to extract features from the raw signal and then an LSTM network to capture the contextual information in the data.

Other works try to solve the emotion recognition task by using facial information with DNNs. For example, Huang et al. [20] proposed a transductive learning framework for image-based emotion recognition by combining DNNs and hypergraphs. More particularly, after the DNN was trained for the emotion classification task, each node in the last fully connected layer was considered as an attribute and used to form a hyperedge in a hypergraph. In another study, Ebrahimi et al. [21] combined CNNs and RNNs to recognize categorical emotions in videos. A CNN was first trained to classify static images containing emotion. Then, the extracted features from the CNN were used to train an RNN to produce an emotion for the whole video.

Combining audio and visual modalities has great success for recognizing emotions. Some studies exploited the beneficial features DNNs can extract [22], [23], [24]. Kim et al. [23] proposed four different DBN architectures with one of them being a basic 2-layer DBN, and the others as a variation of it. The basic architecture first learns the features of the audio and video separately; after which it concatenates these features from the two modalities, it uses them to learn the second layer. The features were evaluated using a Support Vector Machine (SVM). In another study, Kahou et al. [24] proposed to combine modality-specific DNNs to recognize categorical emotions in video. A CNN was used to analyze the video frames, a DBN to capture audio information, a deep autoencoder to model human actions depicted within the entire scene, and finally a CNN network to extract features from the mouth of the human. To output a final prediction they used two techniques that gave similar results. The first is to take the average of modality-specific predictions and in the second they learned an SVM with an RBF kernel using the concatenation features. Another study [25] compared handcrafted features extracted from faces using multi-scale Dense SIFT features (MSDF), and features extracted from CNNs to train linear Support Vector Regression (SVR). The extracted audio features were the extended Geneva Minimalistic Acoustic Parameter Set (eGeMAPS). The combination of the features were used to learn a SVR.

Zhang et al. [26] used a multimodal CNN for classifying emotions with audio and visual modalities. The model is trained in two phases. In the first phase, the two CNNs are pretrained on large image datasets and fine-tuned to perform emotion recognition. The audio CNN takes as input the melspectrogram segment of the audio signal and the video CNN takes the face. In the second phase, a DNN was trained that comprised of a number of fully-connected layers. The concatenation of the features extracted by the two CNNs was the input. In another study, Ringeval et al. [27] use a BLSTMRNN to capture the contextual information that exists in the 
multimodal features (audio, video, physiological) extracted from the data. In a more recent work, Han et al. [28] propose a strength modeling framework, which can be implemented as feature-level and decision-level fusion strategy and comprises of two regression models. The first model's predictions are concatenated with the original feature vector and fed to the second regression model for the final prediction.

The importance of recognizing emotions motivated the creation of the Audio/Visual Emotion Challenge and Workshop (AVEC) [29]. In the 2016 challenge, audio, video and physiological modalities were considered. In one of the submitted models for the challenge, Huang et al. [30] proposed to use variants of a Relevance Vector Machine (RVM) for modeling audio, video and audiovisual data. In another work, a model by Weber et al. [31] used high-level geometry features for predicting dimensional features. Brady et al. [32] also used low- and high-level features for modeling emotions. In a different study, Povolny et al. [33] complemented original baseline features for both audio and video to perform emotion recognition. Somandepalli et al. [34] also used additional features, but only for the audio modality.

All of the works in the literature make use of commonly hand-crafted features in the audio or visual modality or in some cases in both of them. Moreover, they do not always consider temporal information in the data. In this study, we propose a multimodal model trained end-to-end that also considers the contextual temporal information.

\section{Proposed Method}

One of the first steps in a traditional machine learning algorithms is to extract features from the data. To extract features in audio, finite impulse response filters can be used which perform time-frequency decomposition to reduce the influence of background noise [35]. More complicated handengineered kernels, such as gammatone filters [36], which were formulated by studying the frequency responses of the receptive fields of auditory neurons of grass-frogs, can be used as well.

A key component of our model is the convolution operation. For the audio and visual signals, 1-d and 2-d convolution is used, respectively.

$$
(f \star h)(i, j)=\sum_{k=-T}^{T} \sum_{m=-T}^{T} f(k, m) \cdot h(i-k, j-m),
$$

where $f(x)$ is a kernel function whose parameters are learnt from the data of the task in hand. After the spatial-modeling of the signals, which removes background noise and enhances specific parts of the signals for the task in hand, we model the temporal structure of both speech and video by using a recurrent network with LSTM cells. We use LSTM for (i) simplicity, and (ii) to fairly compare against existing approaches which concentrated in the combination of handengineered features and LSTM networks. Finally, our model is subsequently trained with backpropagation by maximizing the concordance correlation loss Equation 3

\section{A. Visual Network}

One of the first steps in the traditional face recognition pipeline is feature extraction utilizing hand-crafted representations such as Scale Invariant Feature Transform (SIFT) and Histogram of Oriented Gradients (HOG). Recently, deep convolutional networks have been used to extract features from faces [26].

In this study, we use a deep residual network (ResNet) of 50 layers [8]. As input to the network we used the pixel intensities from the cropped faces of the subject's video. Deep residual networks adopt residual learning by stacking building blocks of the form:

$$
\mathbf{y}_{k}=\mathcal{F}\left(\mathbf{x}_{k},\left\{W_{k}\right\}\right)+\mathbf{h}\left(x_{k}\right),
$$

where $\mathbf{x}$ and $\mathbf{y}$ are the input and output of the layer $k$, $\mathcal{F}\left(\mathbf{x}_{k},\left\{W_{k}\right\}\right)$ is the residual function to be learned and $h\left(\mathbf{x}_{k}\right)$ can be either an identity mapping or a linear projection to match the dimensions of the function $\mathcal{F}$ and the input $\mathbf{x}$.

The first layer of ResNet-50 is a $7 \times 7$ convolutional layer with 64 feature maps, followed by a max pooling layer of size $3 \times 3$. The rest of the network comprises of 4 bottleneck architectures, where after these architectures a shortcut connection is added. These architectures contain 3 convolutional layers of sizes $1 \times 1,3 \times 3$, and $1 \times 1$, for each residual function. Table I shows the replication and the sizes of the feature maps for each bottleneck architecture. After the last bottleneck architecture, an average pooling layer is inserted.

\begin{tabular}{l|l|l}
\hline \hline Bottleneck layer & Replication & $\begin{array}{l}\text { Number of feature maps } \\
(1 \times 1,3 \times 3, \text { and } 1 \times 1)\end{array}$ \\
\hline 1st & 3 & $64,64,256$ \\
2nd & 4 & $128,128,512$ \\
3rd & 6 & $256,256,1024$ \\
4 th & 3 & $512,512,2048$
\end{tabular}

Table I: The replication of each bottleneck architecture of the ResNet-50 along with the size of the features maps of the convolutions.

\section{B. Speech Network}

In contrast to previous work done in the field of paralinguistics, where acoustic features are first extracted and then passed to a machine learning algorithm, we aim at learning the feature extraction and regression steps in one jointly trained model for predicting the emotion.

Input. We segment the raw waveform to $6 \mathrm{~s}$ long sequences after we preprocess the time-sequences to have zero mean and unit variance to account for variations in different levels of loudness between the speakers. At $16 \mathrm{kHz}$ sampling rate, this corresponds to a 96000-dimensional vector, which is the input to the speech network.

Temporal Convolution. We use $\mathrm{F}=40$ space time finite impulse filters with a $5 \mathrm{~ms}$ window (size of 80) in order to extract finer-scale spectral information from the high sampling rate signal $(16 \mathrm{kHz})$.

Pooling across time. The impulse response of each filter is passed through a half-wave rectifier (analogous to the cochlear 
transduction step in the human ear) and then downsampled to $8 \mathrm{kHz}$ by pooling each impulse response with a pool size $=2$.

Temporal Convolution. We use $\mathrm{M}=40$ space time finite impulse filters of $500 \mathrm{~ms}$ window (size of 4000). These are used to extract more long-term characteristics of the speech and the roughness (i.e., irregularities) of the speech signal.

Max pooling across channels. We perform max-pooling across the channel domain with a pool size of 10 . This reduces the dimensionality of the signal while preserving the necessary statistics of the convolved signal.

Dropout. Due to the large number of parameters compared to the number of training examples, we need to perform some regularization in order for the model not to overfit on the training data. We opt to use dropout with a probability of 0.5 .

\section{Objective function}

To evaluate the agreement level between the predictions of the network and the gold-standard derived from the annotations, the concordance correlation coefficient $\left(\rho_{c}\right)$ [9] has recently been proposed [37], [7]. Nonetheless, previous work minimized the MSE during the training of the networks, but evaluated the models with respect to $\rho_{c}$ [37], [7]. Instead, we propose to include the metric used to evaluate the performance in the objective function $\left(\mathcal{L}_{c}\right)$ used to train the networks. Since the objective function is a cost function, we define $\mathcal{L}_{c}$ as follows:

$$
\begin{aligned}
\mathcal{L}_{c} & =1-\rho_{c}=1-\frac{2 \sigma_{x y}^{2}}{\sigma_{x}^{2}+\sigma_{y}^{2}+\left(\mu_{x}-\mu_{y}\right)^{2}}, \\
& =1-2 \sigma_{x y}^{2} \psi^{-1}
\end{aligned}
$$

where $\psi=\sigma_{x}^{2}+\sigma_{y}^{2}+\left(\mu_{x}-\mu_{y}\right)^{2}$ and $\mu_{x}=\mathbb{E}(\mathbf{x})$, $\mu_{y}=\mathbb{E}(\mathbf{y}), \sigma_{x}^{2}=\operatorname{var}(\mathbf{x}), \sigma_{y}^{2}=\operatorname{var}(y)$ and $\sigma_{x y}^{2}=\operatorname{cov}(\mathbf{x}, \mathbf{y})$. Thus, to minimize $\mathcal{L}_{c}$ (or maximize $\rho_{c}$ ), we backpropagate the gradient of the last layer weights with respect to $\mathcal{L}_{c}$,

$$
\frac{\partial \mathcal{L}_{c}}{\partial \mathbf{x}} \propto 2 \frac{\sigma_{x y}^{2}\left(\mathbf{x}-\mu_{y}\right)}{\psi^{2}}+\frac{\mu_{y}-\mathbf{y}}{\psi},
$$

where all vector operations are done element-wise.

\section{Network Training}

Before training the multimodal network, each modalityspecific network is trained separately to speed up the training procedure.

Visual Network. For the visual network, we chose to finetune the pretrained ResNet-50 on the database used in this work. This model was trained on the ImageNet 2012 [38] classification dataset that consists of 1000 classes. The pretrained model was preferred over training the network from scratch in order to be benefited by the features already learned by the model. To train the network, a 2-layer LSTM with 256 cells each is stack on top of it to capture temporal information.

Speech Network. The CNN network operates on the raw signal to extract features from it. In order to consider the temporal structure of speech, we use two LSTM layers with 256 cells each on top of the CNN.
Multimodal Network. After training the visual and speech networks, the LSTM layers are discarded, and only the extracted features are considered. The speech network extracts 1280-dimensional features while the visual network extracts 2048-dimensional features. These are concatenated to form a 3328-dimensional feature vector and fed to a 2-layer LSTM with 256 cells each. The weights of the LSTM layers are initialized following Glorot based initialization [39], and the visual and speech networks are initialized utilizing the weights of the unimodal models. Finally, the whole network is trained end-to-end. Figure 1 shows the multimodal network.

The goal for each unimodal and the multimodal network is to minimize:

$$
\mathcal{L}_{c}=\frac{\mathcal{L}_{c}^{a}+\mathcal{L}_{c}^{v}}{2},
$$

where $\mathcal{L}_{c}^{a}$ and $\mathcal{L}_{c}^{v}$ are the concordance of the arousal and valence, respectively.

For the recurrent layers of the speech, visual and multimodal networks, we segment the $6 \mathrm{~s}$ sequences to 150 smaller subsequences to match the granularity of the annotation frequency of $40 \mathrm{~ms}$.

\section{DATASET}

A time-continuous prediction of spontaneous and natural emotions (arousal and valence) is investigated on speech and visual data by using the REmote COLlaborative and Affective (RECOLA) database introduced by Ringeval et al. [40]; the full dataset is used for the purpose of this study. Four modalities are included in the corpus: audio, video, electrocardiogram (ECG) and electro-dermal activity (EDA). In total, $9.5 \mathrm{~h}$ of multimodal recordings from 46 French-speaking participants were recorded and annotated for 5 minutes each, performing a collaboration task in dyads during a video conference. Among the participants, 17 were French, three German and three Italian. The dataset is split into three partitions - train (16 subjects), validation (15 subjects) and test (15 subjects) by stratifying (i.e., balancing) the gender and the age of the speakers. Finally, 6 French-speaking annotators (three male, three female) annotated all the recordings.

\section{EXPERIMENTS \& RESULTS}

For training the models, we utilized the Adam optimization method [41], and a fixed learning rate of $10^{-4}$ throughout all experiments. For the audio model, we used a mini-batch of 25 samples. Also, for regularization of the network, we used dropout [42] with $p=0.5$ for all layers except the recurrent ones. This step is important, as our models have a large amount of parameters $(\approx 1.5 M)$ and not regularizing the network makes it prone on overfitting on the training data.

For the video model, the image size used was $96 \times 96$ with a mini-batch of size 2. A small mini-batch is selected because of hardware limitations. The data were augmented by resizing the image to size $110 \times 110$ and randomly cropping it to equal its original size. This produces a scale invariant model. In addition, color augmentation is used by introducing random brightness and saturation to the image. 


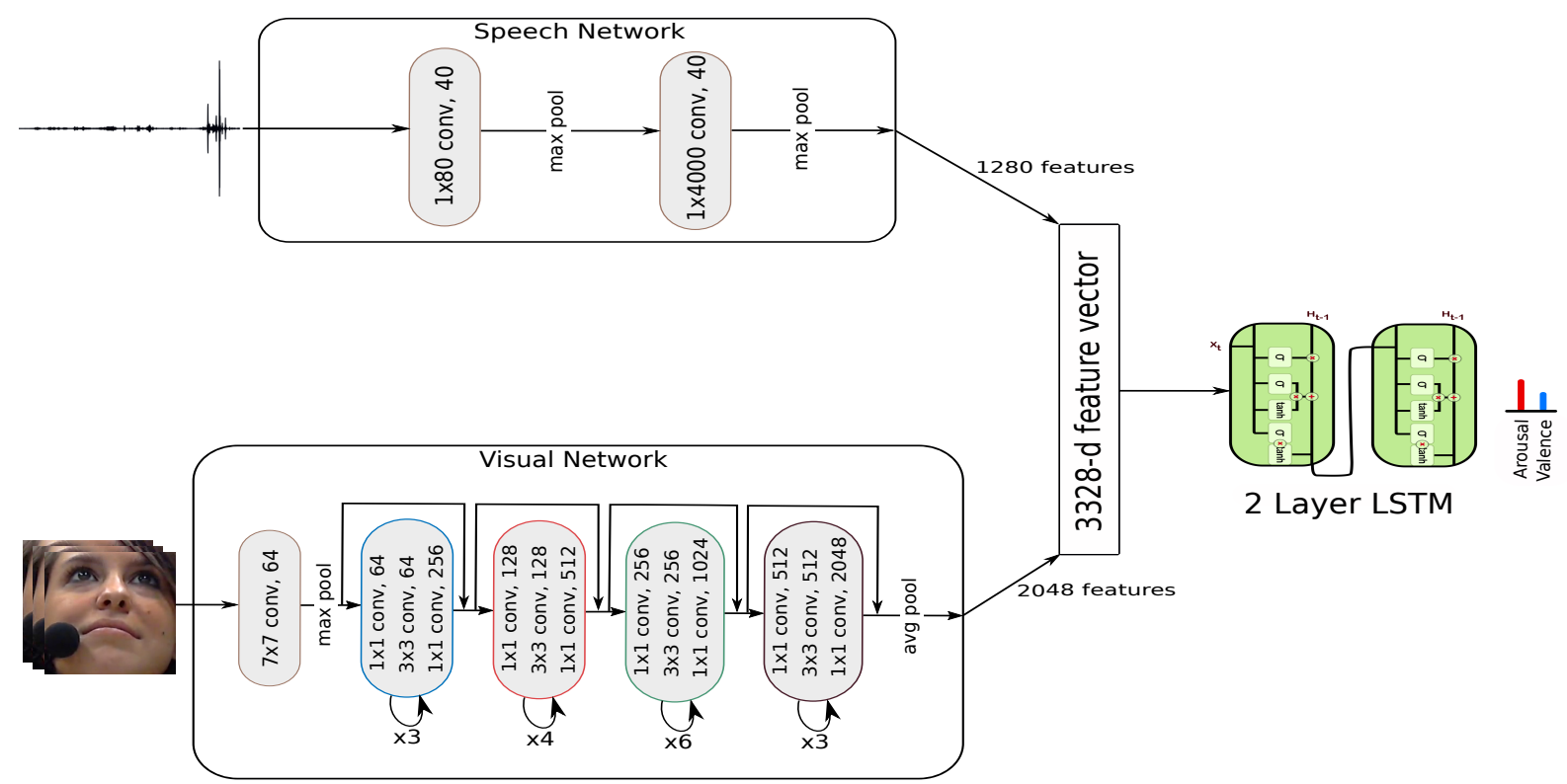

Figure 1: The network comprises of two parts: the multimodal feature extraction part and the RNN part. The multimodal part extracts features from raw speech and visual signals. The extracted features are concatenated and used to feed 2 LSTM layers. These are used to capture the contextual information in the data.

Finally, for all investigated methods, a chain of postprocessing is applied to the predictions obtained on the development set: (i) median filtering (with the size of the window ranging from $0.4 \mathrm{~s}$ to $20 \mathrm{~s}$ ) [7], (ii) centering (by computing the bias between the gold-standard and the prediction) [43], (iii) scaling (using the ratio of standard-deviation of the goldstandard and the prediction as scaling factor) [43] and (iv) time-shifting (by shifting the prediction forward in time with values ranging from $0.04 \mathrm{~s}$ to $10 \mathrm{~s}$ ), to compensate for delays in the ratings [44]. Any of these post-processing steps is kept when an improvement is observed on the $\rho_{c}$ of the validation set, and applied then with the same configuration on the test partition.

\section{A. Ablation study}

Due to memory and training instability concerns [45] it is not always optimal to use very large sequences in recurrent networks. The justification for this can be either the overblowing of gradients or the very deep unrolled graph which makes training of such big networks harder.

In order to choose the best sequence length to feed our LSTM layers, we conducted experiments using sequence lengths of 75, 150, and 300 for both the speech and the visual models. Table II shows the results on the development set. The training for all models lasted 60 epochs.

For the visual network we expect to get the highest value in the valence dimension, while for the speech model in the arousal dimension. Results indicate that the best value for the speech model is 150 while for the visual model it is 300 . Due to the fact that the difference in performance for the visual network is small when a sequence length of 150 or 300 is used, we chose to train the multimodal network with a 150 sequence length.

\begin{tabular}{l|l|l}
\hline \hline $\begin{array}{l}\text { Sequence } \\
\text { length }\end{array}$ & Arousal & Valence \\
\hline \multicolumn{3}{l}{ Visual network } \\
\hline 75 & .293 & .276 \\
150 & .363 & .488 \\
300 & .193 & .496 \\
\hline \multicolumn{3}{l}{ Speech network } \\
\hline 75 & .727 & .345 \\
150 & .744 & .369 \\
300 & .685 & .130
\end{tabular}

Table II: Results (in terms of $\rho_{c}$ ) on arousal and valence after 60 epochs when varying the sequence length for the speech and visual networks.

\section{B. Speech Modality}

Results obtained for each method, using all 46 participants, are shown in Table III. In all of the experiments, our model outperforms the designed features in terms of $\rho_{c}$. One may note, however, that the eGeMAPS [46] feature set provides close performance on valence, which is much more difficult to predict from speech compared to arousal. Furthermore, we show that by incorporating $\rho_{c}$ directly in the optimization function of all networks allows us to optimize the models on the metric $\left(\rho_{c}\right)$ on which we evaluate the models. This provides us with $i$ ) a more elegant way to optimize models, and $\mathrm{ii}$ ) gives consistently better results across all test-runs as seen in Table III 


\begin{tabular}{l|l|l|l}
\hline \hline Predictor & Features & Arousal & Valence \\
\hline \multicolumn{3}{l}{ a. Mean } & squared error objective \\
\hline SVR & eGeMAPS & $.318(.489)$ & $.169(.210)$ \\
SVR & ComParE & $.366(.491)$ & $.180(.178)$ \\
BLSTM & eGeMAPS & $.300(.404)$ & $.192(.187)$ \\
BLSTM & ComParE & $.132(.221)$ & $.117(.152)$ \\
Proposed & raw signal & $.684(.728)$ & $.249(.312)$ \\
\hline \hline \multicolumn{5}{l}{ b. Concordance correlation coefficient objective } \\
\hline BLSTM & eGeMAPS & $.316(.445)$ & $.195(.190)$ \\
BLSTM & ComParE & $.382(.478)$ & $.187(.246)$ \\
\hline Proposed & raw signal & $\mathbf{. 6 9 9}(.752)$ & $\mathbf{. 3 1 1}(.406)$
\end{tabular}

Table III: RECOLA dataset results (in terms of $\rho_{c}$ ) for prediction of arousal and valence. In parenthesis are the performances obtained on the development set. In $a$ ) we optimized the models w.r.t. MSE whereas in $b$ ) w.r.t. $\rho_{c}$.

In addition, we compare the performance on the results obtained for methods that exist in the literature. Most of them have been submitted to the AVEC 2016 challenge, with 27 participants Table IV. For fair comparison, we test our model on the same test set. In case performance on the test or validation set was not reported in the paper, a dash is inserted on. Results show that our model outperforms the other models in the test set when predicting the arousal dimension. It is important to notice that although our model gets a lower $\rho_{c}$ on the arousal dimension for the validation set compared to the baseline of the challenge, its performance is better on the test set.

\begin{tabular}{l|l|l|l}
\hline \hline Predictor & Features & Arousal & Valence \\
\hline Baseline [29] & eGeMAPS & $.648(.796)$ & $\mathbf{. 3 7 5}(.455)$ \\
RVM [30] & eGeMAPS & $-(.750)$ & $-(.396)$ \\
Povolny et al. [33] & Mixed & $-(.833)$ & $-(.503)$ \\
Brady et al. [32] & MFCC & $-(.846)$ & $-(.450)$ \\
Weber et al. [31] & eGeMAPS & $-(.793)$ & $-(.456)$ \\
Somandepalli et al. [34] & Mixed & $-(.800)$ & $-(.448)$ \\
Han et al. [28] & 13 LLDs & $.666(.755)$ & $.364(.476)$ \\
\hline Proposed & raw signal & $\mathbf{. 7 1 5}(.786)$ & $.369(.428)$
\end{tabular}

Table IV: RECOLA dataset results (in terms of $\rho_{c}$ ) for the prediction of arousal and valence using the Speech Network. In parenthesis are the performances obtained on the development set. A dash is inserted if the results were not reported in the original papers.

1) Relation to existing acoustic and prosodic features: The speech signals convey information about the affective state either explicitly, i.e., by linguistic means, or implicitly, i.e., by acoustic or prosodic cues. It is well accepted amongst the research community that certain acoustic and prosodic features play an important role in recognizing the affective state [47. Some of these features, such as the mean of the fundamental frequency (F0), mean speech intensity, loudness, as well as pitch range [46], should thus be captured by our model.

To gain a better understanding of what our speech model learns, and how this relates to existing literature, we study the statistics of gate activations in the network applied on an unseen speech recording. This was accomplished by first finding the three most correlated features from the 256-

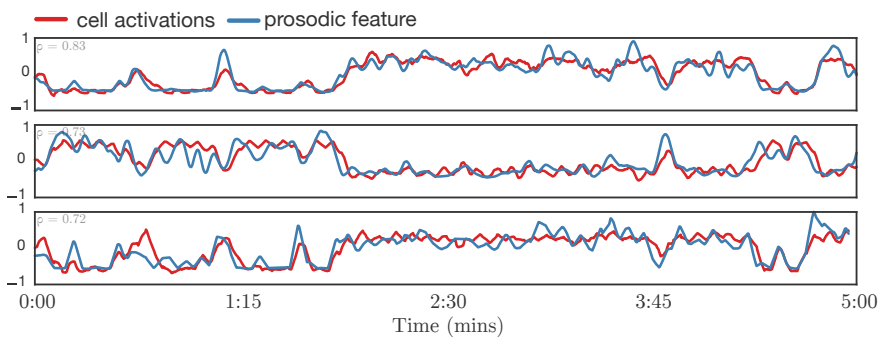

Figure 2: A visualization of three different gate activations vs. different acoustic and prosodic features that are known to affect arousal for an unseen recording to the network. From top to bottom: range of RMS energy ( $\rho=0.83$ ), loudness ( $\rho=$ $0.73)$, mean of fundamental frequency $(\rho=0.72)$

dimensional hidden state vector of the second layer of the recurrent model and the ground truth. Then, we computed the correlation between each of these features with hand-crafted features extracted using the OpenSmile [48] toolkit. We found that our features have a high correlation with the RMS energy, loudness, and mean of fundamental frequency features. A visualization of this correlation is given in Figure 2

\section{Visual Modality}

The visual modality has been shown to more easily predict the valence dimension rather than the arousal. Table V]presents the best results on the RECOLA dataset for the valence dimension. Only the work from Han et al. [28] was not submitted to the AVEC 2016 challenge. The features used for all of the models are appearance and geometric. For appearance, Local Gabor Binary Patterns from Three Orthogonal Planes (LGBPTOP) features were extracted, whereas facial landmarks were extracted for the geometric features. The input to our network are the raw pixel intensities from the face extracted from the frames of the videos using the Multi-Domain Convolutional Neural Network Tracker (MDNet) [49] tracking algorithm. This algorithm takes the bounding box of the face in the first frame of the video and tracks it in all frames.

\begin{tabular}{l|l|l|l}
\hline \hline Predictor & Features & Arousal & Valence \\
\hline Baseline [29] & Appearance & $.343(.483)$ & $.486(.474)$ \\
Baseline [29] & Geometric & $.272(.379)$ & $.507(.612)$ \\
RVM [30] & Geometric & $-(.467)$ & $-(.571)$ \\
RVM [30] & Appearance & $-(.615)$ & $-(.530)$ \\
Video CNN-L4 [33] & Mixed & $-(.595)$ & $-(.497)$ \\
Brady et al [32] & Appearance & $-(.346)$ & $-(.511)$ \\
Weber et al. [31] & Geometric & $-(.476)$ & $-(.683)$ \\
Weber et al. [31] & Appearance & $-(.594)$ & $-(.506)$ \\
Somandepalli et al. [34] & Geometric & $-(.297)$ & $-(.612)$ \\
Somandepalli et al. [34] & Appearance & $-(.481)$ & $-(.474)$ \\
Han et al. [28] & Mixed & $.265(.292)$ & $.394(.592)$ \\
\hline Proposed & raw signal & $\mathbf{. 4 3 5}(.371)$ & $\mathbf{. 6 2 0}(.637)$
\end{tabular}

Table V: RECOLA dataset results (in terms of $\rho_{c}$ ) for prediction of arousal and valence using the Visual Network. In parenthesis are the performances obtained on the development set. A dash is inserted if the results were not reported in the original papers. 


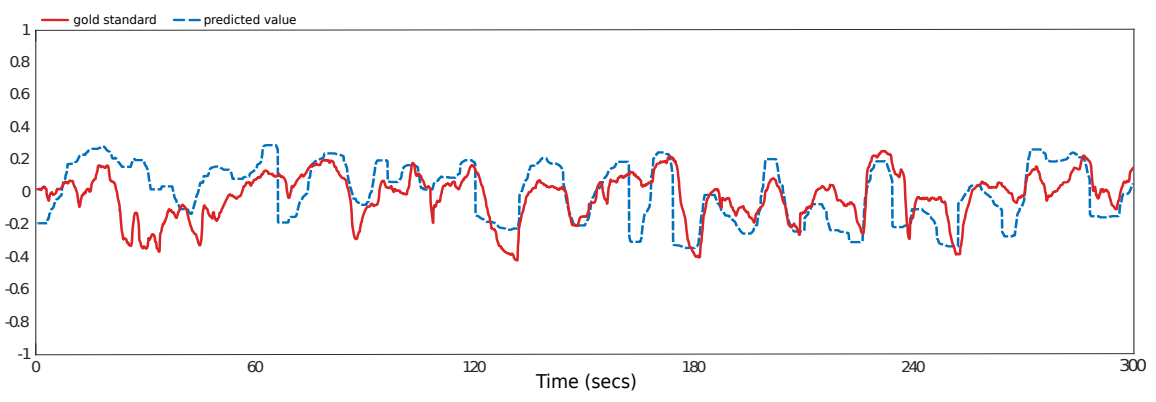

(a) Arousal

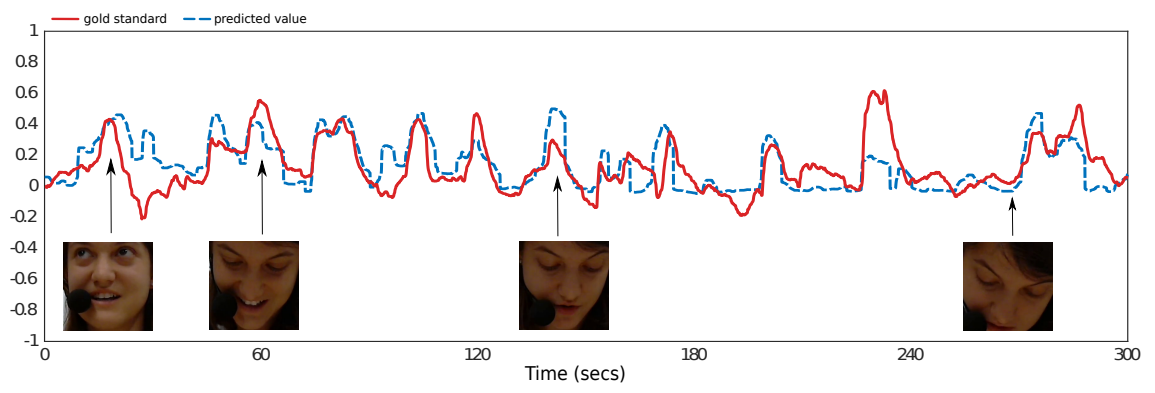

(b) Valence

Figure 3: The predicted and the gold standard for the arousal (a) and valence (b) for the subject with video ID 32, from the test set.

As expected, the visual modality benefits the models in the valence dimension. The only exception is the Video CNNL4 [33] model which performs better in the arousal dimension when appearance features are used. Our model outperforms all the other models in the valence dimension for the test set.

\section{Multimodal Analysis}

Only two other models found in the literature to use both speech and visual modalities on the RECOLA database. These are the Output-Associative Relevance Vector Machine Staircase Regression (OA RVM-SR) [30] and the strength modeling system proposed by Han et al. [28]. To have a fair comparison with the other methods, we utilized both training and validation sets in the training process. We stopped the training of our model when we reached twice the number of epochs required to train the model when only the training set was used. We did this, as the number of both validation and training examples is twice the number of training examples.

Results are shown in Table VI. Our model outperforms the other two models in both the arousal and valence dimensions; especially for the valence dimension with high magnitude. We should also mention here that our system operates directly on the raw pixel domain, while the other two systems made use of a number of geometric features (e.g., 2D/3D facial landmarks etc.) which require the presence of an accurate facial landmark tracking methodology (ours was applied on the results of a conventional face detector only).

To show, however, that our model can also benefit from these features, we incorporate them into our model. In particular, we first extract facial landmarks using the face alignment method by Deng et al [50], and perform Procrustes alignment (ie., removing scale, rotation, translation). Then, we concatenate these features to the feature vector that is input to the recurrent model and we train only the recurrent network while keeping the speech and visual network parameters fixed. Results are depicted in Table VI As expected, these features benefit our model to predict the valence dimension while the arousal is unchanged.

Finally, to further demonstrate the benefits of our model for automatic prediction of arousal and valence, Figure 3 illustrates results for a single test subject from RECOLA.

\begin{tabular}{l|l|l|l|l}
\hline \hline Predictor & $\begin{array}{l}\text { Audio } \\
\text { Features }\end{array}$ & $\begin{array}{l}\text { Visual } \\
\text { Features }\end{array}$ & Arousal & Valence \\
\hline $\begin{array}{l}\text { OA } \\
\text { RVM- } \\
\text { SR }\end{array}$ & $\begin{array}{l}\text { eGeMAPS } \\
\text { ComParE }\end{array}$ & $\begin{array}{l}\text { Geometric } \\
\text { Appearance }\end{array}$ & $.770(.855)$ & $.545(.642)$ \\
\hline Han et al. & 13 LLDs & Mixed & $.610(.728)$ & $.463(.544)$ \\
\hline \hline
\end{tabular}

Table VI: RECOLA dataset results (in terms of $\rho_{c}$ ) for prediction of arousal and valence using the Multimodal Network. In parenthesis are the performance obtained on the development set.

\section{CONCLUSiON}

In this paper, we propose a multimodal system that operates on the raw signal, to perform an end-to-end spontaneous emotion prediction task from speech and visual data. To consider the contextual information, a recurrent network (LSTM) was used. To speed up the training of the model, we pretrained the speech and visual networks, separately. In addition, we 
study the gate activations of the recurrent layers in the speech modality and find cells that are highly correlated with prosodic features that were always assumed to cause arousal. Our experiments on the unimodal modality show that our models achieve significantly better performance on the test set in comparison to other models using the RECOLA database including those submitted to the AVEC2016 challenge, thus demonstrating the efficacy of learning features that better suit the task-at-hand. In addition, our multimodal model greatly outperforms in both the valence and arousal dimensions the other models. Further research on the topic is application of similar architectures for behaviour analysis in the wild.

In future work we aim at incorporating more modalities in our model like physio in order to increase its performance for emotion recognition tasks. In addition, we intend at experimenting with more emotion databases, including ones that provide discrete labels. It would be also interesting to experiment with tasks other than emotion recognition.

\section{ACKNOWLEDGMENTS}

The support of the EPSRC Center for Doctoral Training in High Performance Embedded and Distributed Systems (HiPEDS, Grant Reference EP/L016796/1) is gratefully acknowledged. The work of George Trigeorgis was funded partially from the Google Fellowship in Machine Perception, Speech Technology and Computer Vision. The work of Björn W. Schuller has been partially funded by the EU Horizon 2020 Framework Programme (RIA ARIA VALUSPA, grant no. 645378, IA SEWA \#645094), Stefanos Zafeiriou has been partially funded by the FiDiPro program of Tekes (project number: 1849/31/2015).

\section{REFERENCES}

[1] Q. Ji, Z. Zhu, and P. Lan, "Real-time nonintrusive monitoring and prediction of driver fatigue," IEEE Transactions on Vehicular Technology, vol. 53, no. 4, pp. 1052-1068, 2004.

[2] F. Burkhardt, J. Ajmera, R. Englert, J. Stegmann, and W. Burleson, "Detecting anger in automated voice portal dialogs," in Proceedings of the Annual Conference of the International Speech Communication Association, 2006, pp. 1053-1056.

[3] C.-N. Anagnostopoulos, T. Iliou, and I. Giannoukos, "Features and classifiers for emotion recognition from speech: a survey from 2000 to 2011," Artificial Intelligence Review, vol. 43, no. 2, pp. 155-177, 2015.

[4] G. Hinton, L. Deng, Y. Dong, G. Dahl, A. Mohamed, N. Jaitly, A. Senior, V. Vanhoucke, P. Nguyen, T. Sainath, and B. Kingsbury, "Deep neural networks for acoustic modeling in speech recognition: The shared views of four research groups," Signal Processing Magazine, vol. 29, no. 6, pp. 82-97, November 2012.

[5] A. Graves and N. Jaitly, "Towards end-to-end speech recognition with recurrent neural networks," in Proceedings of International Conference on Machine Learning, Beijing, China, June 2014, pp. 1764-1772.

[6] B. Schuller, S. Steidl, A. Batliner, A. Vinciarelli, K. Scherer, F. Ringeval, M. Chetouani, F. Weninger, F. Eyben, E. Marchi, M. Mortillaro, H. Salamin, A. Polychroniou, F. Valente, and S. Kim, "The interspeech 2013 computational paralinguistics challenge: Social signals, conflict, emotion, autism," in Proceedings of the Annual Conference of the International Speech Communication Association, Lyon, France, August 2013, pp. 148-152.

[7] F. Ringeval, B. Schuller, M. Valstar, S. Jaiswal, E. Marchi, D. Lalanne, R. Cowie, and M. Pantic, "AV+EC 2015 - The First Affect Recognition Challenge Bridging Across Audio, Video, and Physiological Data," in Proceedings of the 5th International Workshop on Audio/Visual Emotion Challenge, Brisbane, Australia, October 2015, pp. 3-8.
[8] K. He, X. Zhang, S. Ren, and J. Sun, "Deep residual learning for image recognition," in Proceedings of the Conference on Computer Vision and Pattern Recognition, Las Vegas, USA, June-July 2016, pp. 770-778.

[9] L. I.-K. Lin, "A concordance correlation coefficient to evaluate reproducibility," Biometrics, vol. 45, no. 1, pp. 255-268, March 1989.

[10] G. Trigeorgis, F. Ringeval, R. Brueckner, E. Marchi, M. A. Nicolaou, B. Schuller, and S. Zafeiriou, "Adieu features? end-to-end speech emotion recognition using a deep convolutional recurrent network," in Proceedings of the IEEE International Conference on Acoustics, Speech and Signal Processing, Shanghai, China, March 2016, pp. 5200-5204.

[11] R. S. Zemel, "Autoencoders, minimum description length and helmholtz free energy," in Proceedings of the Neural Information Processing Systems, Colorado, USA, April 1994, pp. 3-10.

[12] Y. LeCun et al., "Generalization and network design strategies," Connectionism in Perspective, pp. 143-155, 1989.

[13] G. E. Hinton, S. Osindero, and Y.-W. Teh, "A fast learning algorithm for deep belief nets," Neural Computation, vol. 18, no. 7, pp. 1527-1554, 2006.

[14] S. Hochreiter and J. Schmidhuber, "Long short-term memory," Neural Computation, vol. 9, no. 8, pp. 1735-1780, 1997.

[15] J. Ngiam, A. Khosla, M. Kim, J. Nam, H. Lee, and A. Y. Ng, "Multimodal deep learning," in Proceedings of the International Conference on Machine Learning, 2011, pp. 689-696.

[16] D. Hu, X. Li et al., "Temporal multimodal learning in audiovisual speech recognition," in Proceedings of the IEEE Conference on Computer Vision and Pattern Recognition, Las Vegas, USA, June-July 2016, pp. 3574 3582 .

[17] D. Wu, L. Pigou, P.-J. Kindermans, N. D.-H. Le, L. Shao, J. Dambre, and J.-M. Odobez, "Deep dynamic neural networks for multimodal gesture segmentation and recognition," IEEE Transactions on Pattern Analysis and Machine Intelligence, vol. 38, no. 8, pp. 1583-1597, 2016.

[18] K. Han, D. Yu, and I. Tashev, "Speech emotion recognition using deep neural network and extreme learning machine." in Proceedings of the Annual Conference of the International Speech Communication Association, Singapore, September 2014, pp. 223-227.

[19] W. Lim, D. Jang, and T. Lee, "Speech emotion recognition using convolutional and recurrent neural networks," in Proceedings of the Signal and Information Processing Association Annual Summit and Conference, Jeju, Korea, December 2016, pp. 1-4.

[20] Y. Huang and H. Lu, "Deep learning driven hypergraph representation for image-based emotion recognition," in Proceedings of the International Conference on Multimodal Interaction, Tokyo, Japan, November 2016, pp. 243-247.

[21] S. Ebrahimi Kahou, V. Michalski, K. Konda, R. Memisevic, and C. Pal, "Recurrent neural networks for emotion recognition in video," in Proceedings of the International Conference on Multimodal Interaction, Seattle, USA, November 2015, pp. 467-474.

[22] W. Liu, W.-L. Zheng, and B.-L. Lu, "Emotion recognition using multimodal deep learning," in International Conference on Neural Information Processing, Kyoto, Japan, October 2016, pp. 521-529.

[23] Y. Kim, H. Lee, and E. M. Provost, "Deep learning for robust feature generation in audiovisual emotion recognition," in Proceedings of the International Conference on Acoustics, Speech and Signal Processing, Vancouver, Canada, May 2013, pp. 3687-3691.

[24] S. E. Kahou, X. Bouthillier, P. Lamblin, C. Gulcehre, V. Michalski, K. Konda, S. Jean, P. Froumenty, Y. Dauphin, N. BoulangerLewandowski et al., "Emonets: Multimodal deep learning approaches for emotion recognition in video," Journal on Multimodal User Interfaces, vol. 10, no. 2, pp. 99-111, 2016.

[25] B. Sun, S. Cao, L. Li, J. He, and L. Yu, "Exploring multimodal visual features for continuous affect recognition," in Proceedings of the 6th International Workshop on Audio/Visual Emotion Challenge, Amsterdam, The Netherlands, October 2016, pp. 83-88.

[26] S. Zhang, S. Zhang, T. Huang, and W. Gao, "Multimodal deep convolutional neural network for audio-visual emotion recognition," in Proceedings of the International Conference on Multimedia Retrieval, New York, USA, June 2016, pp. 281-284.

[27] F. Ringeval, F. Eyben, E. Kroupi, A. Yuce, J.-P. Thiran, T. Ebrahimi, D. Lalanne, and B. Schuller, "Prediction of asynchronous dimensional emotion ratings from audiovisual and physiological data," Pattern Recognition Letters, vol. 66, no. Supplement C, pp. 22-30, 2015.

[28] J. Han, Z. Zhang, N. Cummins, F. Ringeval, and B. Schuller, "Strength modelling for real-world automatic continuous affect recognition from audiovisual signals," Image and Vision Computing, vol. 65, no. Supplement C, pp. 76 - 86, 2017.

[29] M. Valstar, J. Gratch, B. Schuller, F. Ringeval, D. Lalanne, M. Torres Torres, S. Scherer, G. Stratou, R. Cowie, and M. Pantic, "Avec 2016: 
Depression, mood, and emotion recognition workshop and challenge," in Proceedings of the 6th International Workshop on Audio/Visual Emotion Challenge, Amsterdam, The Netherlands, October 2016, pp. 3-10.

[30] Z. Huang, B. Stasak, T. Dang, K. Wataraka Gamage, P. Le, V. Sethu, and J. Epps, "Staircase regression in oa rvm, data selection and gender dependency in avec 2016," in Proceedings of the 6th International Workshop on Audio/Visual Emotion Challenge, Amsterdam, The Netherlands, October 2016, pp. 19-26.

[31] R. Weber, V. Barrielle, C. Soladié, and R. Séguier, "High-level geometrybased features of video modality for emotion prediction," in Proceedings of the 6th International Workshop on Audio/Visual Emotion Challenge, Amsterdam, The Netherlands, October 2016, pp. 51-58.

[32] K. Brady, Y. Gwon, P. Khorrami, E. Godoy, W. Campbell, C. Dagli, and T. S. Huang, "Multi-modal audio, video and physiological sensor learning for continuous emotion prediction," in Proceedings of the 6th International Workshop on Audio/Visual Emotion Challenge, Amsterdam, The Netherlands, October 2016, pp. 97-104.

[33] F. Povolny, P. Matejka, M. Hradis, A. Popková, L. Otrusina, P. Smrz, I. Wood, C. Robin, and L. Lamel, "Multimodal emotion recognition for avec 2016 challenge," in Proceedings of the 6th International Workshop on Audio/Visual Emotion Challenge, Amsterdam, The Netherlands, October 2016, pp. 75-82.

[34] K. Somandepalli, R. Gupta, M. Nasir, B. M. Booth, S. Lee, and S. S. Narayanan, "Online affect tracking with multimodal kalman filters," in Proceedings of the International Workshop on Audio/Visual Emotion Challenge, New York, USA, October 2016, pp. 59-66.

[35] H. Hirsch, P. Meyer, and H. Ruehl, "Improved speech recognition using high-pass filtering of subband envelopes," in EUROSPEECH, Genoa, Italy, September 1991, pp. 413-416.

[36] R. Schlüter, L. Bezrukov, H. Wagner, and H. Ney, "Gammatone features and feature combination for large vocabulary speech recognition," in Proceedings of the IEEE International Conference on Acoustics, Speech and Signal Processing, Honolulu, USA, April 2007, pp. 649-652.

[37] F. Ringeval et al., "Prediction of asynchronous dimensional emotion ratings from audiovisual and physiological data," Pattern Recognition Letters, vol. 66, pp. 22-30, November 2015.

[38] O. Russakovsky, J. Deng, H. Su, J. Krause, S. Satheesh, S. Ma, Z. Huang, A. Karpathy, A. Khosla, M. Bernstein et al., "Imagenet large scale visual recognition challenge," International Journal of Computer Vision, vol. 115, no. 3, pp. 211-252, 2015.

[39] X. Glorot and Y. Bengio, "Understanding the difficulty of training deep feedforward neural networks," in Proceedings of the Thirteenth International Conference on Artificial Intelligence and Statistics, Sardinia, Italy, May 2010, pp. 249-256.

[40] S.-A. S. J. Ringeval, Fabien and D. Lalanne, "Introducing the RECOLA Multimodal Corpus of Remote Collaborative and Affective Interactions," in Proceedings of the IEEE International Conference and Workshops on Automatic Face and Gesture Recognition, Shanghai, China, April 2013, pp. $1-8$.

[41] D. Kingma and J. Ba, "Adam: A method for stochastic optimization," arXiv preprint arXiv:1412.6980, 2014.

[42] N. Srivastava, G. Hinton, A. Krizhevsky, I. Sutskever, and R. Salakhutdinov, "Dropout: A simple way to prevent neural networks from overfitting," Journal of Machine Learning Research, vol. 15, no. 1, pp. 19291958, January 2014.

[43] M. Kächele, P. Thiam, G. Palm, F. Schwenker, and M. Schels, "Ensemble methods for continuous affect recognition: Multimodality, temporality, and challenges," in Proceedings of the 5th International Workshop on Audio/Visual Emotion Challenge, Brisbane, Australia, October 2015, pp. 9-16.

[44] S. Mariooryad and C. Busso, "Correcting time-continuous emotional labels by modeling the reaction lag of evaluators," IEEE Transactions on Affective Computing, vol. 6, no. 2, pp. 97-108, April-June 2015.

[45] K. Cho, B. Van Merriënboer, C. Gulcehre, D. Bahdanau, F. Bougares, H. Schwenk, and Y. Bengio, "Learning phrase representations using rnn encoder-decoder for statistical machine translation," arXiv preprint arXiv:1406.1078, 2014.

[46] F. Eyben, K. R. Scherer, B. W. Schuller, J. Sundberg, E. André, C. Busso, L. Y. Devillers, J. Epps, P. Laukka, S. S. Narayanan et al., "The geneva minimalistic acoustic parameter set (gemaps) for voice research and affective computing," IEEE Transactions on Affective Computing, vol. 7, no. 2, pp. 190-202, 2016.

[47] K. Scherer, "Vocal communication of emotion: A review of research paradigms," Speech Communication, vol. 40, no. 1-2, pp. 227-256, April 2003.

[48] F. Eyben, M. Wöllmer, and B. Schuller, "Opensmile: the munich versatile and fast open-source audio feature extractor," in Proceedings of the 18th ACM international conference on Multimedia, Firenze, Italy, October 2010, pp. 1459-1462.

[49] H. Nam and B. Han, "Learning multi-domain convolutional neural networks for visual tracking," in Proceedings of the IEEE Conference on Computer Vision and Pattern Recognition, Las Vegas, USA, June 2016, pp. 4293-4302.

[50] Z. Y. Deng J., Trigeorgis G. and Z. S., "Joint multi-view face alignment in the wild," arXiv preprint arXiv:1708.06023, 2017. 


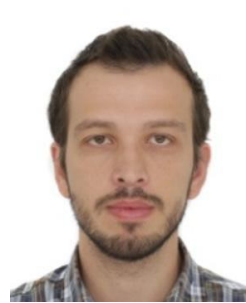

Panagiotis Tzirakis is a first year $\mathrm{PhD}$ student in the High Performance Embedded and Distributed Systems (HiPEDS) Center for Doctoral Training (CDT) program of Imperial College London. His research interests are in the areas of deep learning, face identification, and speech recognition.

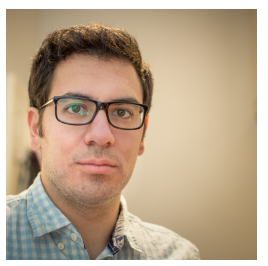

George Trigeorgis is currently a $\mathrm{Ph} . \mathrm{D}$. student at the Department of Computing, Imperial College London. He has received an MEng in Artificial Intelligence in 2013 from the Imperial College London. He was the recipient of the prestigious Google Doctoral Fellowship n Machine Perception, Speech Technology and Computer Vision. During the course of his Ph.D. he has published in the top venues for machine learning and perception such as CVPR, ICML, NIPS, and T-PAMI.

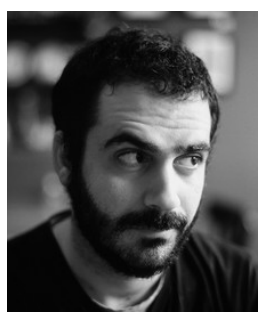

Mihalis A. Nicolaou is a Lecturer at the Department of Computing at Goldsmiths, University of London and an Honorary Research Fellow with the Department of Computing at Imperial College London. Mihalis obtained his $\mathrm{PhD}$ from the same department at Imperial, while he completed his undergraduate studies at the Department of Informatics and Telecommunications at the University of Athens, Greece. Mihalis' research interests span the areas of machine learning, computer vision and affective computing. He has been the recipient of several awards and scholarships for his research, including a Best Paper Award at IEEE FG, while publishing extensively in related prestigious venues. Mihalis served as a Guest Associate Editor for the IEEE Transactions on Affective Computing and is a member of the IEEE.

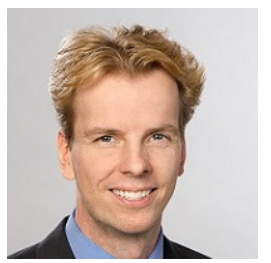

Björn W. Schuller received his diploma, doctoral degree, habilitation, and Adjunct Teaching Professorship all in EE/IT from TUM in Munich/Germany. At present, he is a Reader (Associate Professor) in Machine Learning heading GLAM - the Group on Language, Audio \& Music at Imperial College London/UK, Full Professor and Chair of Embedded Intelligence for Health Care and Wellbeing at the University of Augsburg/Germany, and the cofounding CEO of audEERING GmbH. Previously, he was chair of Complex \& Intelligent (2014 2017) and Sensor Systems (2013) at the University of Passau/Germany and headed the Machine Intelligence and Signal Processing Group at TUM (2006 - 2014). In 2013 he was also invited as a permanent Visiting Professor at the Harbin Institute of Technology/P.R. China and the University of Geneva/Switzerland. In 2012 he was with Joanneum Research in Graz/Austria remaining an expert consultant. In 2011 he was guest lecturer in Ancona/Italy and visiting researcher in the Machine Learning Research Group of NICTA in Sydney/Australia. From 2009 to 2010 he was with the CNRS-LIMSI in Orsay/France, and a visiting scientist at Imperial College. He co-authored $600+$ technical contributions $(16,000+$ citations, h-index $=62)$ in the field.

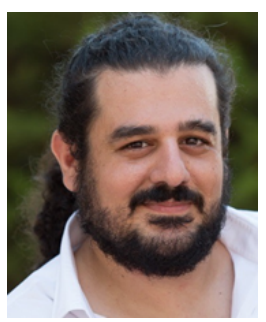

Stefanos Zafeiriou (M'09) is currently a Reader in Machine Learning and Computer Vision with the Department of Computing, Imperial College London, London, U.K, and a Distinguishing Research Fellow with University of Oulu under Finish Distinguishing Professor Programme. He was a recipient of the Prestigious Junior Research Fellowships from Imperial College London in 2011 to start his own independent research group. He was the recipient of the President's Medal for Excellence in Research Supervision for 2016. He has received various awards during his doctoral and post-doctoral studies. He currently serves as an Associate Editor of the IEEE Transactions on Affective Computing and Computer Vision and Image Understanding journal. In the past he held editorship positions in IEEE Transactions on Cybernetics the Image and Vision Computing Journal. He has been a Guest Editor of over six journal special issues and co-organised over 13 workshops/special sessions on specialised computer vision topics in top venues, such as CVPR/FG/ICCV/ECCV (including three very successfully challenges run in ICCV' 13 , ICCV' 15 and CVPR' 17 on facial landmark localisation/tracking). He has co-authored over 55 journal papers mainly on novel statistical machine learning methodologies applied to computer vision problems, such as 2-D/3-D face analysis, deformable object fitting and tracking, shape from shading, and human behaviour analysis, published in the most prestigious journals in his field of research, such as the IEEE T-PAMI, the International Journal of Computer Vision, the IEEE T-IP, the IEEE T-NNLS, the IEEE T-VCG, and the IEEE T-IFS, and many papers in top conferences, such as CVPR, ICCV, ECCV, ICML. His students are frequent recipients of very prestigious and highly competitive fellowships, such as the Google Fellowship x2, the Intel Fellowship, and the Qualcomm Fellowship x3. He has more than 4500 citations to his work, h-index 36 . He is the General Chair of BMVC 2017. 